\title{
Contact temperature measurement by infrared thermography during resistance spot welding process
}

\author{
by E. Geslain*, T. Pierre*, P. Rogeon, C. Pouvreau and L. Cretteur* \\ * Univ. Bretagne Sud, UMR CNRS 6027, IRDL, 56100 Lorient, France, edouard.geslain@univ-ubs.fr \\ ** ArcelorMittal Global R\&D, 1 route de Saint-Leu, BP 30109, F-60761 Montataire Cedex, France
}

\begin{abstract}
During a resistance spot weld, a current of several kiloamperes passes through a stack of sheets clamped between two electrodes. We are interested here in the very first moments of the welding, when the combined effects of electrical and thermal contact resistances at sheet/sheet $(\mathrm{S} / \mathrm{S})$ and electrode/sheet $(\mathrm{E} / \mathrm{S})$ interfaces are at the origin of intense overheating. In order to estimate the contact temperatures, non-contact infrared thermography measurements coupled with an instrumentation by micro-thermocouples welded on the sheets are performed. The evolution of the emissivity is measured and the temperatures at the interfaces are evaluated.
\end{abstract}

\section{Introduction}

In the automotive application, a solution for the reduction of the energy consumption is the development of lightweight vehicles. The trend is to reduce the steel sheet thickness by using different steel grades (low carbon steel, high strength steel, press hardened steel...) with different coatings. These sheets are mainly joined by resistance spot welding process. This process, widely used in this industrial field, is fast, easily automated, and economic. The assemblies used for the manufacture of the body in white include two or three sheets.

During a resistance spot weld, a current of several kiloamperes passes through a stack of steel sheets clamped between two electrodes in copper alloy. The combined effects of electrical and thermal contact resistances at E/S and S/S interfaces are at the origin of intense ephemera heating that occur at the beginning of welding, which influence strongly the initiation and the growing of the nugget [1]. The welding of two sheets is generally without issues. In the case of a dissymmetric assembly of three sheets including a very thin low carbon steel sheet as external sheet, the nugget may not develop sufficiently in the thin sheet [1]. The understanding of the phenomena at the interfaces would be improved with the knowledge of the local temperature. However, the microscopic dimensions of the interfacial zones associated with very fast response times, about ten microseconds, make their instrumentation with micro-sensors very difficult.

Classically, the geometrical configuration of resistance spot welding does not allow any direct observations of phenomena at the interfaces during the process. Some authors proposed to use half-electrodes and deport the welding process at the edge of the stacks [2]. Combining this method with an infrared imaging [3] of the edge, this paper deals with the evolution of the temperatures at the interfaces of assemblies of three sheets, heterogeneous in grade, thickness, and coatings, at the very first moments of the process (between room temperature and $600{ }^{\circ} \mathrm{C}$ ). A high-resolution ( $15 \mu \mathrm{m} /$ pitch) fast infrared camera is used, and for the implementation of this method, two different assemblies are tested. In order to estimate the true temperatures at the interfaces, the non-contact measurements by infrared thermography are coupled with an instrumentation by micro-thermocouples implanted on the sheets in order to measure at first the emissivity and to deduce the interface temperature.

\section{Description of the experimental device}

\subsection{Presentation of the samples}

Fig. 1 presents the two assemblies instrumented with micro-thermocouples: a symmetrical stack-up (on the left) and a dissimilar stack-up (on the right). The composition of the sheets are the following:

- $\quad$ the DP600 sheet is an advanced high strength steel (AHSS) with zinc coating and $1.47 \mathrm{~mm}$ thickness;

- the Usibor 1500 is a $1.2 \mathrm{~mm}$ thickness press hardened steel (PHS) for hot stamped, protected on both sides by an aluminum-silicon (Al-Si) coating;

- $\quad$ the AM54 sheet is a zinc coating low carbon steel (LCS) for deep drawing. It is a very thin sheet of $0.57 \mathrm{~mm}$ thick with zinc coating.

On the assemblies, two perpendicular axis are considered, an axis $y$ coinciding with the axis of symmetry for the electrodes and an axis $x$, parallel to the sheet surfaces, between the two thick sheets. The icons $\bullet$ represent $50 \mu \mathrm{m}$ thermocouples for which the junction of the two wires is about $130 \mu \mathrm{m}$. Four thermocouples are welded, two on the DP600 sheets (Tc12 and Tc21), one at the interfaces (Tc11), and one on Usibor $\AA$ sheet (Tc22). 

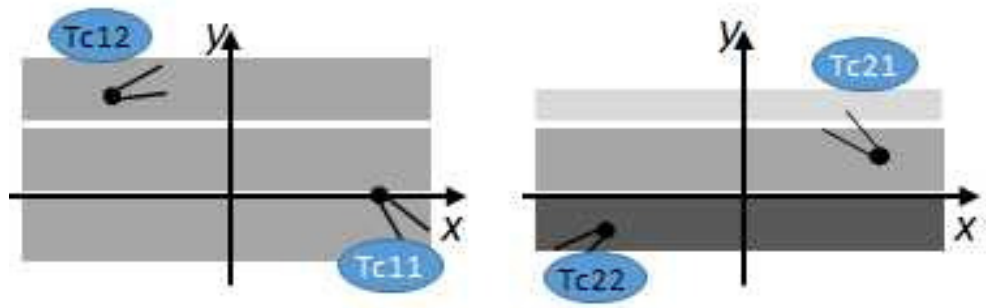

Fig. 1. Sketch of the two assemblies from symmetrical (left) to very dissymmetrical (right), instrumented with $50 \mu \mathrm{m}$ thermocouples $(\bullet)$

The steel sheet edges have been sandblasted at first, which gives a homogeneous surface aspect of the assemblies. The emissivity has been measured in our laboratory and is equal to 0.63 . The half-electrodes have been covered by a high temperature black paint.

\subsection{The welding device}

Fig. 2 presents a global view of the experimental apparatus composed of the resistance spot welding process: a GYSPOT MFDC welding machine, a data recorder and the infrared camera, which is fixed above the welding electrodes as shown in Fig. 3.

The welding parameters are the same for each assembly: $200 \mathrm{~ms}$ of welding time, $2 \mathrm{kN}$ of welding force, and a $4.5 \mathrm{kA}$ current intensity. The data recorder with $1 \mathrm{kHz}$ speed records thermocouples values, the current intensity, and the voltage between electrodes. This last value is also measured on the IR camera to synchronize measurements.

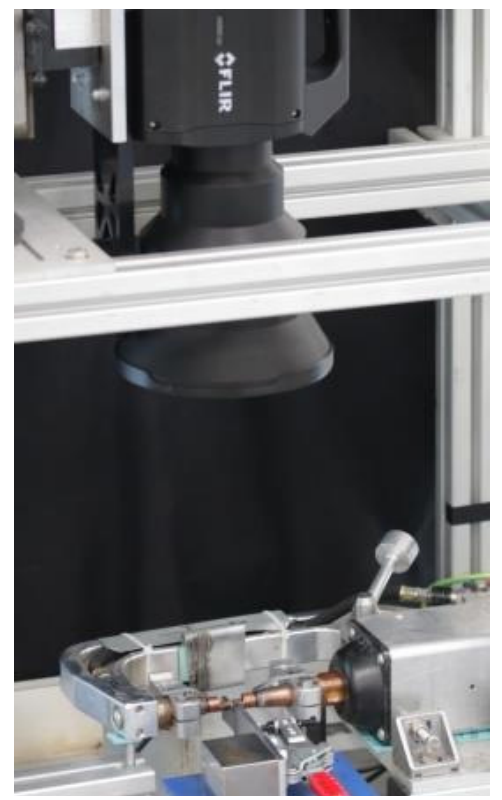

Fig.2: Picture of the experimental device.

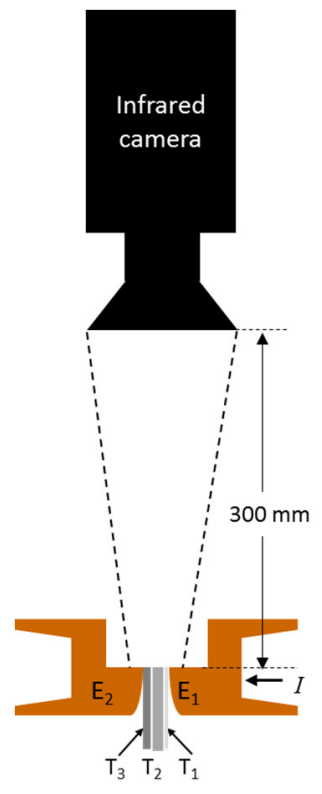

Fig. 3. Scheme of the experimental device.

\subsection{The infrared camera}

The infrared camera is a FLIR X6580sc with a microscopic lens. This microscopic lens has a long focal distance $(300 \mathrm{~mm})$ and a sapphire window to protect him. An optical filter reduces the wavelength range between $4.9 \mu \mathrm{m}$ and $5.1 \mu \mathrm{m}$. The operating temperature range is then $\left[20^{\circ} \mathrm{C}-600{ }^{\circ} \mathrm{C}\right]$ for a blackbody emissivity. On this range, three integration times are necessary:

- $\quad$ preset 0: $325 \mu \mathrm{s}$ from $20^{\circ} \mathrm{C}$ to $170{ }^{\circ} \mathrm{C}$;

- $\quad$ preset 1: $100 \mu \mathrm{s}$ from $86^{\circ} \mathrm{C}$ to $282^{\circ} \mathrm{C}$;

- $\quad$ preset 2: $10 \mu \mathrm{s}$ from $260{ }^{\circ} \mathrm{C}$ to $600{ }^{\circ} \mathrm{C}$. 
The IR camera gives information expressed either in terms of counts $D L$ (digital level) or in terms of temperature $T$ (expressed in kelvin) with the help of an appropriate calibration. The counts are considered here, where they are classically related with the effective emissivity $\varepsilon(T)$ with a function given by Eq. (1), where $C(T)$ is a Planck's law type function (2) [4].

$$
\begin{gathered}
D L=\varepsilon(T) C(T)+[1-\varepsilon(T)] C\left(T_{e n v}\right) \\
C(T)=\frac{R}{e^{\frac{B}{T}}-F}
\end{gathered}
$$

In Eq. (1), the terms on the right correspond to the radiosity: the first one is the proper emission of the investigated surface of emissivity $\varepsilon(T)$ and the second one is the reflected part of the radiative flux coming from the environment at temperature $T_{\text {env. }}$

The IR camera has been calibrated for this specific experiment with a blackbody. The three coefficients in Eq. (2), $R, B$, and $F$ have been estimated through ordinary least-squares. Fig. 4 presents the evolution of the experimental counts (squares) versus the blackbody temperature for the three integration times and the fitted calibration curves (solid and dashed lines) given by Eq. (1). It is noticeable that the preset 2 has less sensitivity than the two others.

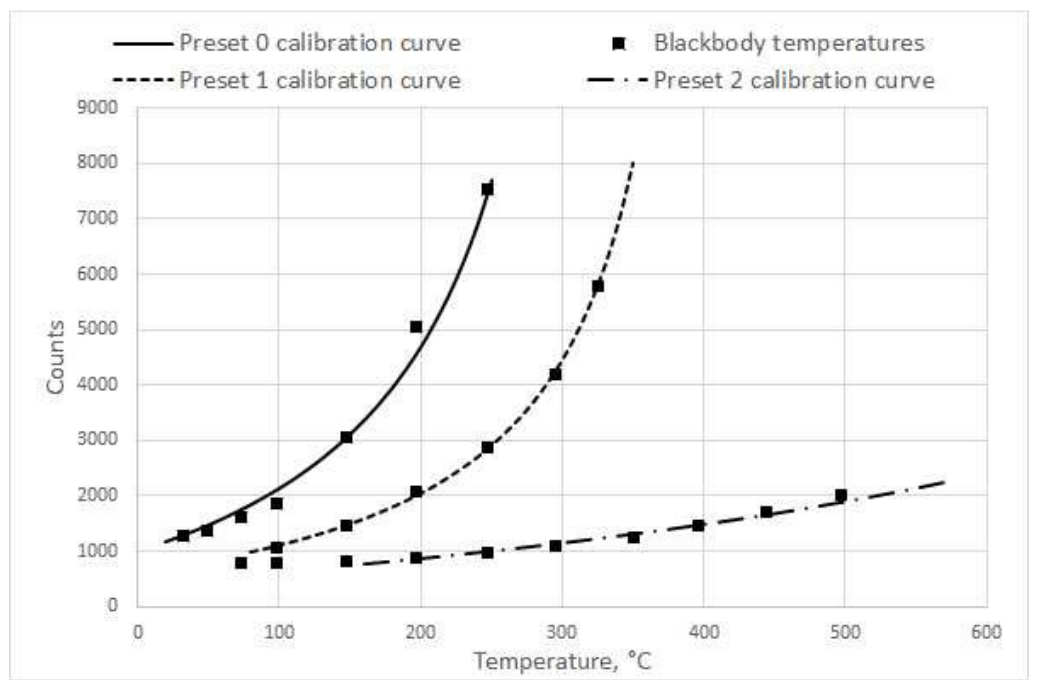

Fig. 4: Fitting of the calibration curves (solid and dashed lines) on the blackbody experimental temperatures (squares) for the calibration of the IR camera for the three integration times (see section 2).

The quality of the estimation has been studied with the help of the covariance matrix (3), which allows to determine the correlation between the three parameters. To start estimation, all values are initially put to 1 . It shows that, for preset 2 , the coefficient $F$ is the best estimated since it is less correlated with the other ones. The coefficients $R$ and $B$ seem correlated. However, even these parameters are correlated, the best criterion is the residuals between the estimated and the theoretical counts, which average value must be as weak as possible, as presented in Fig. 4. For the three integration times, all results in terms of variance and residuals are gathered in Table 1.

$$
\left[\begin{array}{ccc}
\sigma_{R}^{2} & \sigma_{R B} & \sigma_{R F} \\
\sigma_{B R} & \sigma_{B}^{2} & \sigma_{B F} \\
\sigma_{F R} & \sigma_{F B} & \sigma_{F}^{2}
\end{array}\right]
$$


Table 1. Calibration parameters.

\begin{tabular}{|c|c|c|c|c|c|c|c|c|c|}
\hline Integration time & \multicolumn{3}{|c|}{$325 \mu \mathrm{s}$} & \multicolumn{3}{|c|}{$100 \mu \mathrm{s}$} & \multicolumn{3}{|c|}{$10 \mu \mathrm{s}$} \\
\hline Covariance & $\sigma_{R B}$ & $\sigma_{R F}$ & $\sigma_{B F}$ & $\sigma_{R B}$ & $\sigma_{R F}$ & $\sigma_{B F}$ & $\sigma_{R B}$ & $\sigma_{R F}$ & $\sigma_{B F}$ \\
\hline Correlation coefficient & 1 & 1 & 1 & 1 & 1 & 1 & 0.994 & 0.631 & 0.591 \\
\hline Residuals & \multicolumn{3}{|c|}{$-6 \pm 76$} & \multicolumn{3}{|c|}{$-13 \pm 101$} & \multicolumn{3}{|c|}{$-5 \pm 45$} \\
\hline$R$ & \multicolumn{3}{|c|}{977.058} & \multicolumn{3}{|c|}{76.829} & \multicolumn{3}{|c|}{16.630} \\
\hline$B$ & \multicolumn{3}{|c|}{245.497} & \multicolumn{3}{|c|}{50.376} & \multicolumn{3}{|c|}{12.148} \\
\hline$F$ & \multicolumn{3}{|c|}{1.472} & \multicolumn{3}{|c|}{1.075} & \multicolumn{3}{|c|}{1.007} \\
\hline
\end{tabular}

\section{The sheets emissivity measurements}

The experiments have been performed for the two stacks of steel sheets combining the IR camera and the thermocouple measurements. Unfortunately, temperature acquisitions with Tc11, Tc21 failed (Fig. 1). Only the temperatures from the Tc12 and Tc22 thermocouples are available and exploitable.

The principle of the IR investigation from the thermograms is to place a cursor (+) on the image to get the experimental counts $D L$ and to estimate the emissivity with Eq. (4), where $T$ is the temperature given by the thermocouples.

$$
\varepsilon(T)=\frac{D L-C\left(T_{e n v}\right)}{C(T)-C\left(T_{e n v}\right)}
$$

However, the problem lays on the knowledge of the counts where the thermocouples are set, since their emissivity is different from the steel sheets' emissivity. Even if the presence of invasive thermocouples can provoke heat losses in its vicinity by fin effect, we assume that the thermocouples tip behaves as a lumped body and that its response time shows no delay compared to the inertia of the thermal phenomena [5]. Thus, the idea is to consider four cursors around the thermocouple cursor, such as presented in Figs. 5 and 6. Therefore, the counts $D L_{i, j}$ (located at $i$ along the $x$-direction and at $j$ along the $y$-direction) on the steel sheet just underneath the thermocouple is equal to the average of the counts located in the vicinity $(i \pm 1$ and $j \pm 1)$ of the tip:

$$
D L_{i, j} \approx \frac{D L_{i-1, j}+D L_{i+1, j}+D L_{i, j-1}+D L_{i, j+1}}{4}
$$

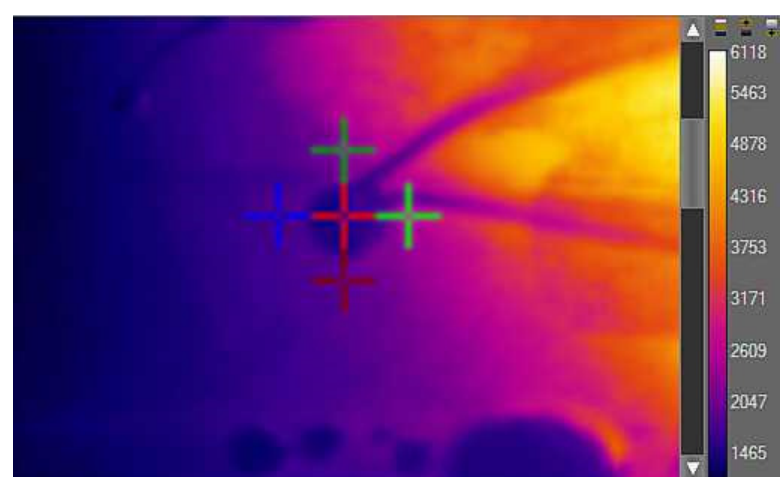

Fig. 5: IR image with the five cursors placed on and around the thermocouple tip.

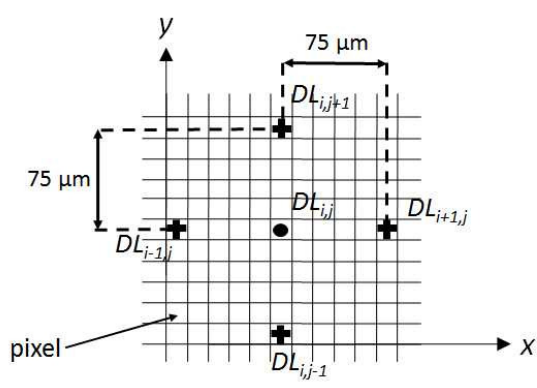

Fig. 6: Example of illustration of the four cursors (+)

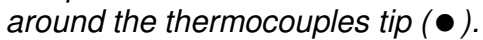

Table 2 presents an example of experimental results of counts, thermocouple temperatures, and emissivity versus time for Tc12 placed on the first symmetrical assembly and for the integration time of $10 \mu \mathrm{s}$. The calculated mean count $D L_{i, j}$ underneath the thermocouple Tc12 is estimated, which allows us to consider it as the count emitted by the steel sheet itself. The evolution of $D L_{i, j}$ and of the thermocouple temperatures $T$ for the three assemblies are plotted in Fig. 7.

The emissivity uncertainty $\Delta \varepsilon(T)$ (5) in Table 2 is evaluated as the systematic error from Eqs. (1) and (2), depending on $D L_{i, j}, C\left(T_{\text {env }}\right)$, and $T$. 


$$
\Delta \varepsilon(T)=\left|\frac{1}{C(T)-C\left(T_{e n v}\right)}\right| \Delta D L_{i, j}+\left|\frac{D L_{i, j}-C(T)}{\left[C(T)-C\left(T_{e n v}\right)\right]^{2}}\right| \Delta C\left(T_{e n v}\right)+\left|\frac{B C(T) e^{\frac{B}{T}}}{T^{2}\left(e^{\frac{B}{T}}-F\right)} \frac{D L_{i, j}-C\left(T_{e n v}\right)}{\left[C(T)-C\left(T_{e n v}\right)\right]^{2}}\right| \Delta T
$$

Table 2: Experimental results of counts and thermocouple temperatures in the case for Tc12 at the integration time of $10 \mu \mathrm{s}$, where $C\left(T_{\text {env }}\right)=767$ is the noise level.

\begin{tabular}{|c|c|c|c|c|c|c|c|c|c|}
\hline $\boldsymbol{t}(\mathbf{m s})$ & $\boldsymbol{T}_{\boldsymbol{c}}\left({ }^{\circ} \mathbf{C}\right)$ & $\boldsymbol{C}(\boldsymbol{T})$ & $\boldsymbol{D} \boldsymbol{L}_{i, j}$ & $\begin{array}{c}\Delta \boldsymbol{D} \boldsymbol{L}_{i, j} \\
(\%)\end{array}$ & $\boldsymbol{D} \boldsymbol{L}_{i-1, j}$ & $\boldsymbol{D} L_{i+1, j}$ & $D L_{i, j-1}$ & $\boldsymbol{D} L_{i, j+1}$ & $\varepsilon \pm \Delta \varepsilon$ \\
\hline 0 & $21.9 \pm 0.1$ & $\begin{array}{c}767 \\
\text { (noise) }\end{array}$ & $767 \pm 1$ & 0.2 & 768 & 766 & 767 & 766 & - \\
\hline 5.964 & $21.7 \pm 0.1$ & 767 & $769 \pm 2$ & 0.2 & 767 & 769 & 770 & 768 & - \\
\hline 11.928 & $21.1 \pm 0.1$ & 767 & $772 \pm 3$ & 0.4 & 777 & 773 & 775 & 771 & - \\
\hline 17.893 & $27.7 \pm 0.1$ & 767 & $778 \pm 4$ & 0.5 & 787 & 776 & 782 & 780 & - \\
\hline 23.857 & $28.9 \pm 0.1$ & 767 & $789 \pm 6$ & 0.7 & 814 & 785 & 795 & 795 & - \\
\hline 29.821 & $42.1 \pm 0.1$ & 767 & $818 \pm 14$ & 1.7 & 861 & 805 & 826 & 837 & - \\
\hline 35.785 & $102.3 \pm 0.1$ & 767 & $861 \pm 26$ & 3.0 & 861 & 836 & 868 & 900 & - \\
\hline 41.750 & $277.0 \pm 0.1$ & 1054 & $907 \pm 42$ & 4.6 & 909 & 868 & 915 & 971 & $0.49 \pm 0.16$ \\
\hline 47.714 & $394.4 \pm 0.1$ & 1484 & $973 \pm 63$ & 6.5 & 986 & 918 & 976 & 1069 & $0.29 \pm 0.10$ \\
\hline 53.678 & $505.9 \pm 0.1$ & 2040 & $1050 \pm 89$ & 8.4 & 1075 & 972 & 1048 & 1184 & $0.22 \pm 0.09$ \\
\hline 59.642 & $608.9 \pm 0.1$ & 2762 & $1138 \pm 115$ & 10.1 & 1181 & 1035 & 1128 & 1310 & $0.18 \pm 0.07$ \\
\hline 65.607 & $713.1 \pm 0.1$ & 3846 & $1255 \pm 147$ & 11.7 & 1339 & 1121 & 1231 & 1460 & $0.16 \pm 0.06$ \\
\hline 71.571 & $813.4 \pm 0.1$ & 5543 & $1371 \pm 181$ & 13.2 & 1479 & 1214 & 1332 & 1626 & $0.13 \pm 0.05$ \\
\hline 77.535 & $904.5 \pm 0.1$ & 7687 & $1509 \pm 222$ & 14.8 & 1651 & 1322 & 1453 & 1817 & $0.10 \pm 0.04$ \\
\hline
\end{tabular}

At first, from Table 2, Fig. 7 plots the evolution of the counts and the temperature of Tc12 versus time, and Fig. 8 the evolution of the calculated emissivity versus the temperature for the three presets 0,1 , and 2 presented in section 2 . For presets 0 and 1, only three emissivities could have been calculated and more with preset 2 .

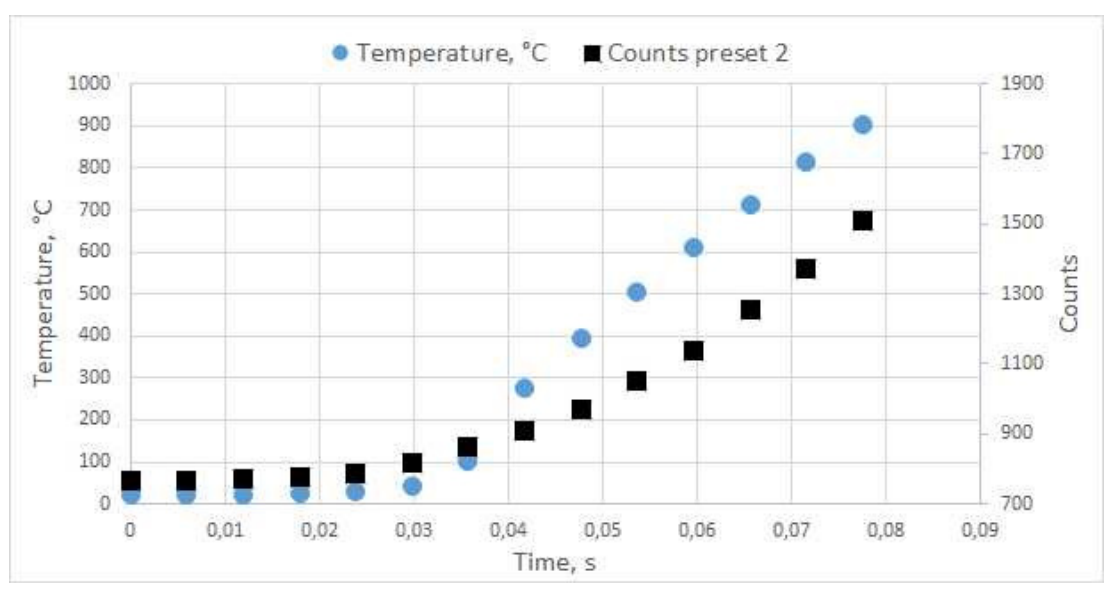

Fig. 7. Example of counts and thermocouple temperature Tc12 versus time for the first assembly with three similar steel sheets.

Generally, Fig. 8 shows that the calculated emissivities for the three presets present progressive decreasing evolutions. Even if the emissivity increases when the temperature decreases, the emissivities seem to tend toward the initial value of 0.6 measured initially at room temperature. 


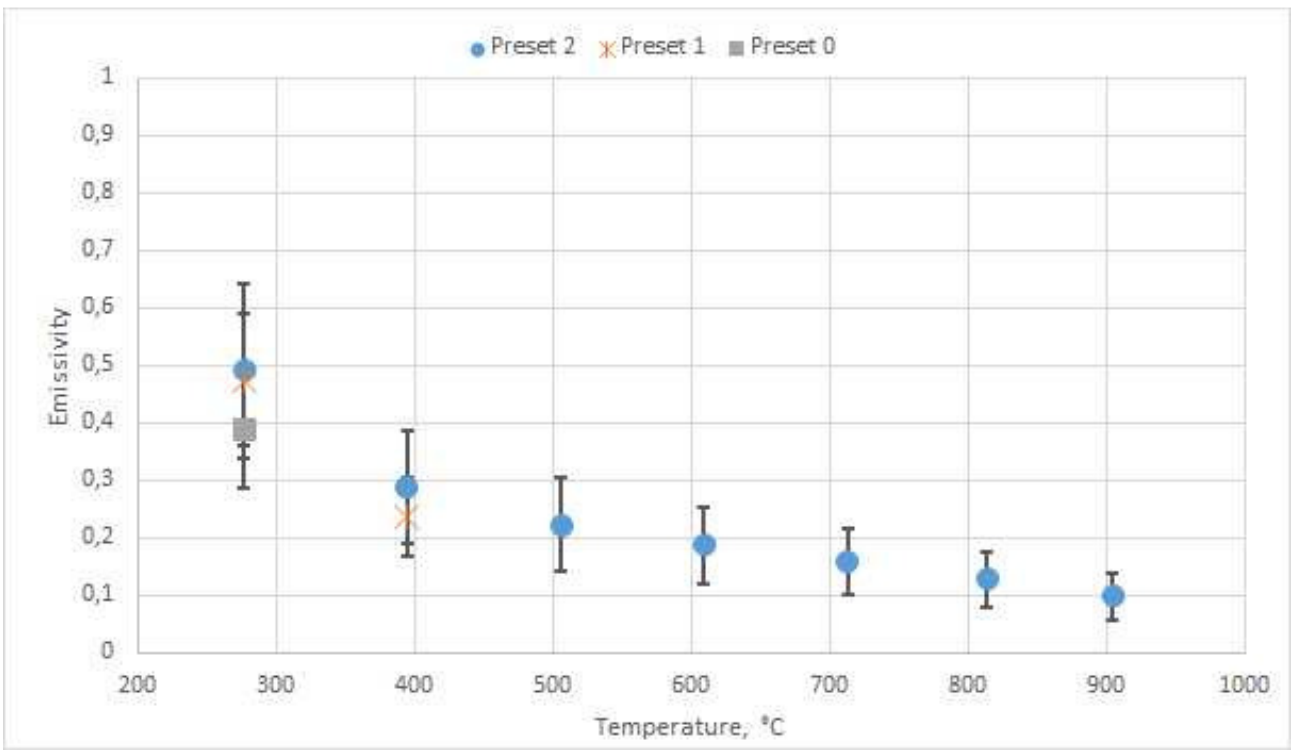

Fig. 8. Estimation of the emissivity for the three presets versus the temperature given by the thermocouple Tc12.

The same procedure is done with thermocouple temperature Tc22. Results are presented and compared with the previous ones in Fig. 9. According to the discrepancy expressed by the vertical error bars, the calculated emissivities in both cases show close results versus temperature. This is also consistent with the fact that the initial aspect of the assembly edge is homogeneous.

Literature presents generally an increase of the emissivity with the temperature due to, for example, oxidation problem [6]. In the case of the RSW, the process time lasts less than $100 \mathrm{~ms}$, which makes us suppose that this phenomenon has not the time to develop. The weak calculated emissivity values is consistent with the materials emissivity, which decreases strongly with temperature when the materials reach the melting point [7].

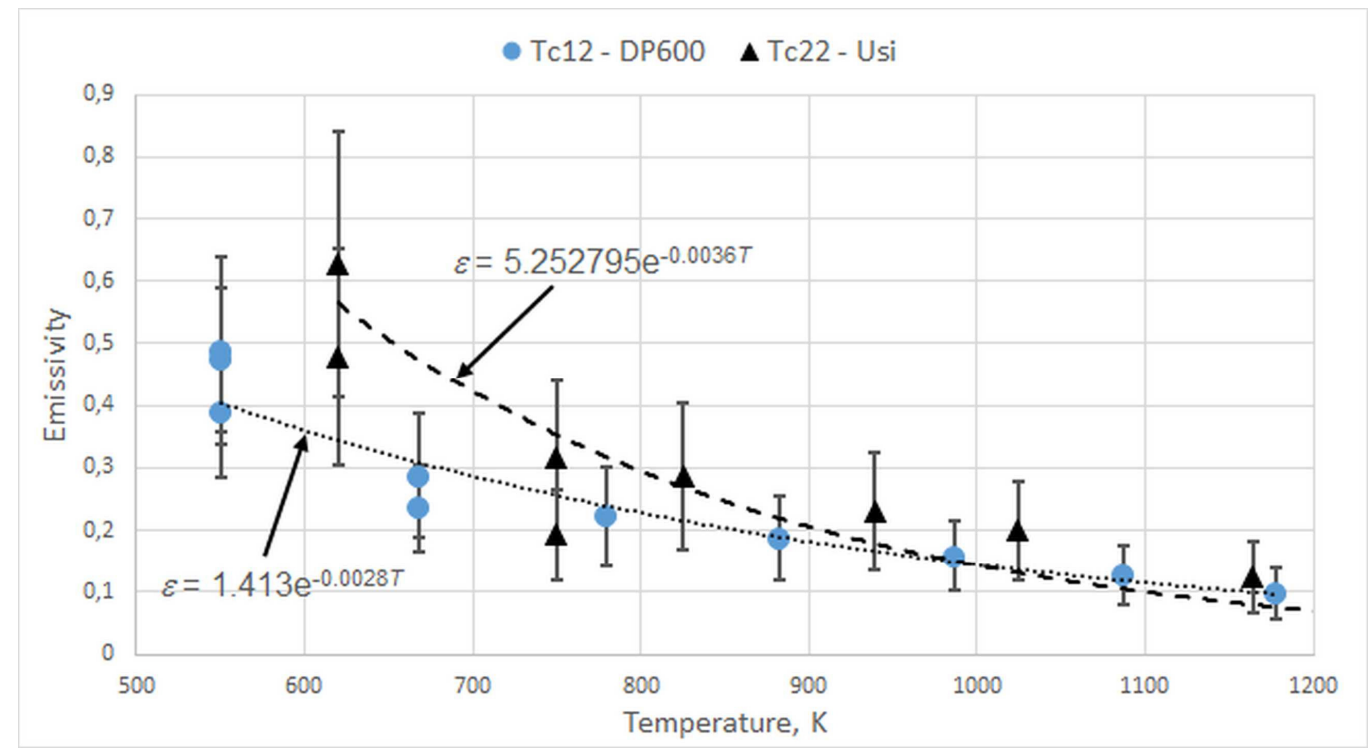

Fig. 9. Estimation of the emissivities for the three presets versus the temperature given by the thermocouple Tc 12 and Tc22.

Two exponential models (6) are fitted on the calculated emissivities, where $a_{0}$ and $a_{1}$ are coefficients estimated by ordinary least squares and $T$ the temperature expressed in kelvin to be consistent with the temperature of Eq. (2).

$$
\mathcal{E}^{\text {estim }}(T)=a_{0} e^{-a_{1} T}
$$


These models are then used to estimate the temperature in the close vicinity of the interfaces of the second dissymmetrical assembly.

\section{Contact temperature measurements}

Once the emissivity is known on the edge of the steel sheet, the temperature at the interfaces of the second assembly versus time is calculated thanks to several cursors placed along y-axis perpendicular to the interfaces (Fig. 10):

- $\quad$ cursors 1, 2, and 3 concern the electrode (Cu)/AM54 interface;

- $\quad$ cursors 4, 5, and 6 concern the AM54/DP600 interface;

- $\quad$ cursors 7, 8, and 9 concern the DP600/Usibor® interface;

- and cursors 10,11 , and 12 concern the Usibor $\AA /$ electrode $(\mathrm{Cu})$ interface.

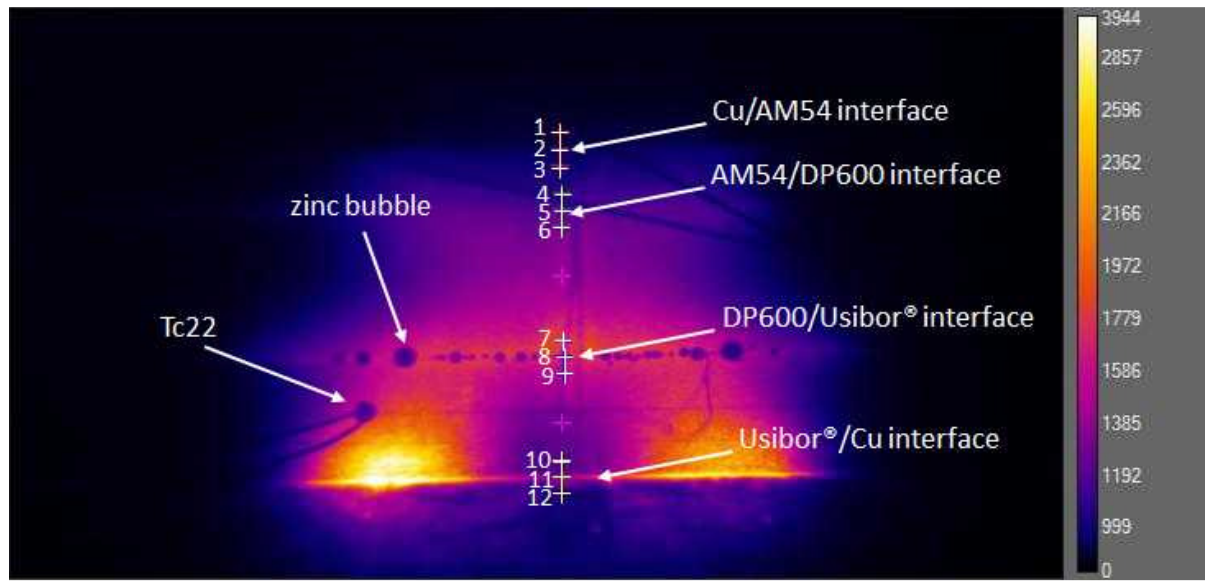

Fig. 10: IR thermogram of the dissymmetrical assembly and position of the cursors in the vicinity of every interfaces at the time $t=35 \mathrm{~ms}$.

The counts $D L$ are recorded with the preset 2 versus the time and the temperatures $T$ are calculated by minimizing the Eq. (7) using $\varepsilon^{\text {estim }}$ defined by Eq. (6) and obtained from Tc12 (Fig. 9). The minimization of (7) is performed with the help of the fsolve Matlab function using Levenberg-Marquardt algorithm.

$$
D L-\left\{\varepsilon^{e s t i m}(T)\left[C(T)-C\left(T_{e n v}\right)\right]+C\left(T_{e n v}\right)\right\} \rightarrow 0
$$

Only the estimated temperatures at the steel sheets interfaces are presented, that is to say, for the cursors 4 to 9. Results of estimation are plotted in Fig. 11. First, it shows that the AM54/DP600 interface heats less than the DP600/Usibor ${ }^{8}$ one. This is due to the fact that the thermal and electrical contact resistances at the AM54/DP600 interface with the Zn coating are much weaker than that at the DP600/Usibor® interface with the AISi coating [1, 8].

This is mainly at the DP600/Usibor ${ }^{\circledR}$ interface that the heating of the assembly initiates. The three temperatures at the DP600/Usibor ${ }^{\circledR}$ interface seem evolving similarly versus time. Nevertheless, the cursor 8 at the interface shows a faster increase since it is placed where the thermal and electrical contact resistances initiate important overheating and thus high temperatures.

An observation can be made at $t=35 \mathrm{~ms}$ for the cursor 8 in Fig. 11. There is a slight plateau at the temperature of around $410{ }^{\circ} \mathrm{C}$. The IR thermogram at this moment allows to see the presence of a zinc bubble, such as presented in Fig. 10. Indeed, as written in section 2, the AM54 steel sheet possesses a thin zinc coating, which melts at $420{ }^{\circ} \mathrm{C}$. Considering the uncertainty on the emissivity value, the estimated temperature corresponding to the moment when the bubble appears is in rather good agreement with the melting temperature. 


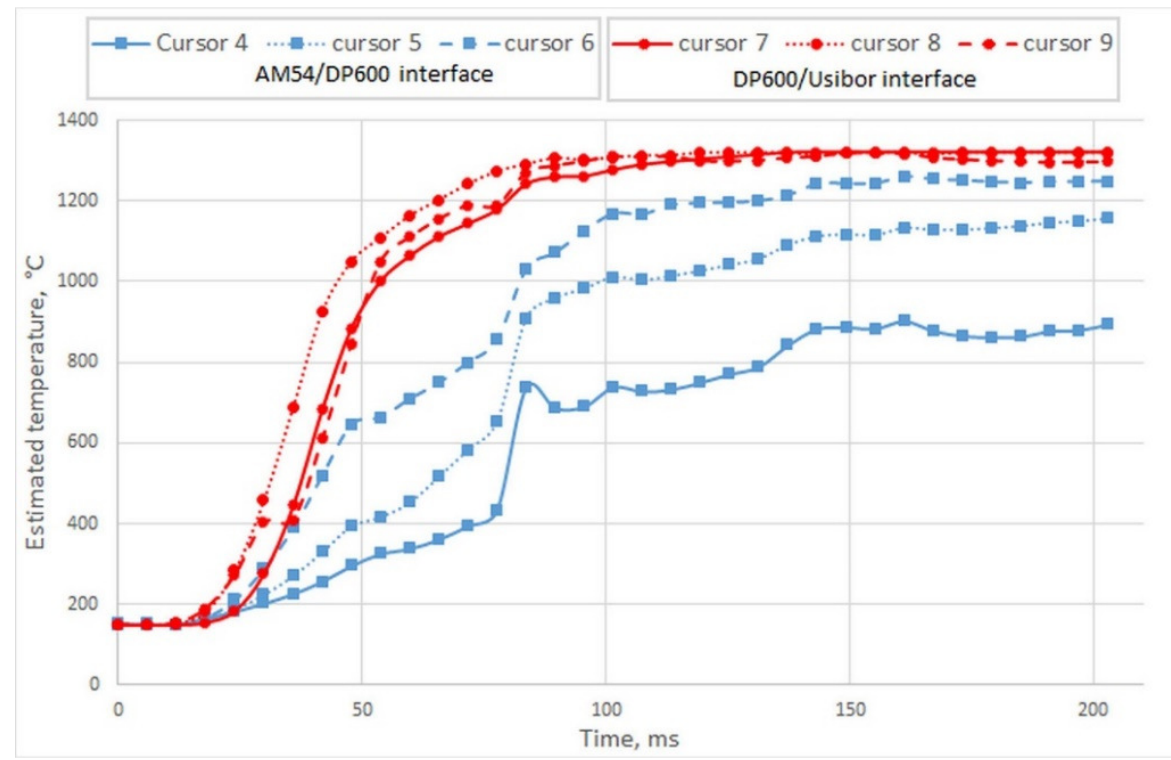

Fig. 11: Evolution of the estimated temperatures close to and at the interfaces versus time in the case of the dissymmetrical assembly.

\section{Conclusion}

This study aims to improve the understanding of the difficulties encountered during the resistance spot welding process of a dissymmetrical assembly with three different steel sheets. In situ instrumentation with micro-thermocouples has been performed and the welding scene recorded by a fast and high-resolution infrared camera. Estimation results of the emissivity and of the temperature at the interfaces between the sheets of the stack have been proposed. The calculated emissivities and their uncertainties of the steel sheets are rather consistent with the literature for this type of materials. The high rises of the estimated temperatures at the interfaces with the Usibor® sheet confirm the role of the strong values of electrical and thermal contact resistances induced by the AISi coating.

\section{Acknowledgments}

The authors would like to thank Mr Laurent Cretteur and the ArcelorMittal Company for the steels supply and the funding of this study. The authors would also like to thanks Mr Barré from the GYS society for his implication regarding the welding machine modifications.

\section{REFERENCES}

[1] E. Geslain, P. Rogeon, T. Pierre, C. Pourvreau, L. Cretteur, Coating effects on contact conditions in resistance spot weldability, Journal of Materials Processing Tech., 253 (2018), 160-167.

[2] R. Ikeda, Y. Okita, M. Ono, K. Yasuda, T. Terasaki, "Development of advanced resistance spot welding process using control of electrode force and welding current during welding", Weld. Int., vol. 28, no. 1, 2014.

[3] Füssel, V. Wesling, A. Voigt, E. C. Klages, Visualisierung der Temperaturentwicklung in der SchweiSzone einschlieSlich der SchweiSelektroden über den gesamten zeitlichen Verlauf eines PunktschweiSprozesses, Schweiss. und Schneid., 64 (2012), 634-642.

[4] R. Frappier, A. Benoit, P. Paillard, T. Baudin, R. Le Gall, T. Dupuy, Quantitative infrared analysis of welding processes: temperature measurement during RSW and CMT-MIG welding, Science Technology of Welding and Joining, 19-1 (2014), 38-43.

[5] A. Terzis, J. von Wolfensdorf, B. Weigand, P. Ott, Thermocouple thermal inertia effects on impingement heat transfer experiments using the transient liquid crystal technique, Meas. Sci. Technol, 23, 2012, 115303 (13pp).

[6] Y.S. Touloukian, Thermal radiative properties, Plenum, New York, 1970.

[7] H. Watanabe, M. Susa, H. Fukuyama, K. Nagata, Phase (Liquid/Solid) Dependence of the Normal Spectral Emissivity for Iron, Cobalt, and Nickel at Melting Points, International Journal of Thermophysics, Vol. 24, No. 2, March 2003.

[8] E. Geslain, Soudage par résistance des tôles fines revêtues : formation du noyau dans un assemblage de trois tôles, PhD thesis, Université de Bretagne-Sud, 2018. 